(C) 2017 IEEE. Personal use of this material is permitted. Permission from IEEE must be obtained for all other uses, in any current or future media, including reprinting/republishing this material for advertising or promotional purposes, creating new collective works, for resale or redistribution to servers or lists, or reuse of any copyrighted component of this work in other works. 


\section{Multi-Period Modeling of Behind-the-Meter Flexibility}

\author{
Rui Pinto, Manuel A. Matos \\ INESC TEC and Faculty of Engineering of the University of \\ Porto \\ Porto, Portugal \\ rbpinto@inesctec.pt,mam@fe.up.pt
}

\author{
Ricardo J. Bessa, José Gouveia, Clara Gouveia \\ INESC TEC \\ Porto, Portugal \\ ricardo.j.bessa@inesctec.pt, jose.m.gouveia@inesctec.pt, \\ clara.s.gouveia@inesctec.pt
}

\begin{abstract}
Reliable and smart information on the flexibility provision of Home Energy Management Systems (HEMS) represents great value for Distribution System Operators and Demand/flexibility Aggregators while market agents. However, efficiently delimiting the HEMS multi-temporal flexibility feasible domain is a complex task. The algorithm proposed in this work is able to efficiently learn and define the feasibility search space endowing DSOs and aggregators with a tool that, in a reliable and time efficient faction, provides them valuable information. That information is essential for those agents to comprehend the fully grid operation and economic benefits that can arise from the smart management of their flexible assets. House load profile, photovoltaic (PV) generation forecast, storage equipment and flexible loads as well as pre-defined costumer preferences are accounted when formulating the problem. Support Vector Data Description (SVDD) is used to build a model capable of identifying feasible HEMS flexibility offers. The proposed algorithm performs efficiently when identifying the feasibility of multi-temporal flexibility offers.
\end{abstract}

Index Terms-HEMS multi-temporal flexibility, support vector data description, energy storage.

\section{INTRODUCTION}

The noteworthy proliferation of Distributed Renewable Energy Sources (DRES) in Low Voltage (LV) and Medium Voltage (MV) distribution grids is creating technical difficulties to the Distribution System Operator (DSO) which are prone to increase as DRES becomes more and more present in those grids supported by the price decrease in the Photovoltaic (PV) technology [1], [2]. With significant levels of PV integration within LV and MV conventional grids it is expected that problems related with violation of bus voltage limits arise especially at times of high PV production. Microgrids (MGs) are expected to allow for a more flexible operation by taking advantage of the embedded flexible loads, storage equipment and their intelligent management by means of Home Energy Management Systems (HEMS) combined with smart meters.

From the DSO perspective, microgrids are valuable assets regarding their contribution for the system operation. The flexibility to be provided by microgrids and multi-microgrids systems [3] can be used for voltage control features at the $\mathrm{MV} / \mathrm{LV}$ substation [4], for power losses reduction and to maximize DRES penetration and hosting capacity, which might translate into lesser environmental impacts and financial gains for prosumers. In the near future, significant low carbon technologies like small-scale embedded generators, energy storage, heat-pumps, electric-water heaters or even electricvehicles will certainly be common in residential buildings. This will allow for a greater operation flexibility by means of demand response programs, residential load aggregators working in the markets or even individual HEMS maximizing customers profits by providing flexibility capacity to meet the DSO operational needs [5]-[7].

Aiming at maximizing the penetration of DRES while maintaining the regulated levels of system's reliability and quality of service new management/operation tools for the distribution grids will have to be developed which must include different types of flexibility provided by active demand management and storage units [8]. Having to account for all these new features, the problem formulation of typical DSO operational exercises, such as Optimal Power Flow (OPF) routines, increases dramatically when storage units are accounted, as inter-temporal constraints must be present when evaluating the flexibility to be provided by such equipment.

This work pretends to develop an algorithm that models the HEMS multi-period flexibility, namely flexible loads such as Electric Water Heaters (EWH), as well as microgeneration sources (e.g., PV) and domestic storage units, and offers to the DSO the flexibility that can be provided. In this methodology, HEMS feasible flexibility provision offers for a certain number of periods ahead are computed and then used as training set in a Support Vector Data Description function. After that, the multi-temporal flexibility provision domain can be delimited. With this approach, reduced data regarding the HEMS flexibility provision potential is passed to the DSO. This way, the OPF complexity from the DSO perspective can be reduced as the HEMS internal equipment does not need to be modelled. This procedure can be repeatedly done as updated information becomes available, in a sliding-window mechanism. Additionally, current Demand Response aggregators or future flexibility market agents bidding in grid ancillary services markets can used the proposed tool as the microgrid grid services provision becomes more commonly accepted and used. Having the HEMS multi-period flexibility search space 
defined aggregators can improve the estimation of the flexibility that their assets are able to deliver. In a strong market environment, reliable and optimally acquired information regarding the maximum range of flexibility provision can have significant value for such agents. The remaining of the paper is organized as follows: chapter II refers to the state of the art on DRES flexibility modeling; in chapter III the methodology adopted in this study is detailed, presenting the different steps of the algorithm being proposed; chapter IV presents the case study; and finally in chapter $\mathrm{V}$ the main conclusions and future work lines are presented.

\section{STATE OF THE ART}

There are recent and rather few research work focusing this area of HEMS flexibility provision [9]-[14]. In [9], Zhao et al. propose a geometric approach to aggregate flexibility modeling of thermostatically controlled loads (TCL). The set of admissible power profiles of an individual TCL is shown to be a polytope, and their aggregated flexibility is the Minkowski sum of the individual polytopes. To cope with computational burden, the authors adopt several approximations regarding the calculation of the Minkowski sums, the modeling of temperature evolution of the TCL, and by assuming a continuous power input of the TCLs instead of the actual binary nature. In this work, the modeling of aggregated TLCs for flexibility definition is addressed without considering the joint provision capacity that can arise from TCLs and storage units together within the HEMS concept.

Polymeneas and Meliopoulos [10] aimed at derive an aggregated model of a distribution system composed by responsive distributed energy resources without including the modeling of individual devices. The main purpose was to approximate the variations in the feasible set as function of feeder consumption. To achieve that, the authors used a timemoving ellipsoid to model those variations. Despite the computational burden decreases when aggregated models of the distribution system are used, there are no guarantees that the active and reactive power targets defined are feasible and whether or not it can be assigned to the actual devices in the system.

Supported by the systems operators' need to correctly evaluate and plan ahead the flexibility adequacy of power systems so that a feasible and economical operation is guaranteed, Nosair et al. [13] proposed flexibility envelopes to describe the flexibility potential of power systems and its individual resources. The authors study the intra-hourly output variation of DRES. An envelope that encompasses all possible intra-hourly deviation and variation of the RES is proposed while considering that for a certain sub-hourly time there is maximum output variability. By plotting the $95 \%$ percentile of the probability distribution of all the sub-hourly time steps produces an envelope which encompasses the majority of realizations of reserve ramping requirements for that hour and DRES. A similar process is proposed to define the flexibility envelope of each power system resource. HEMS are not considered neither are the resource owners operation preferences for their flexibility assets accounted when defining the flexibility envelopes.

\section{METHODOLOGY}

\section{A. The concept of multi-period flexibility}

Accounting for the house inflexible demand, PV forecasts, expected hot water demand, State of Charge (SoC) of the storage units, and the temperature of the water in the EWH tank, a set of feasible trajectories related to the HEMS operating point can be constructed and correspond to a discrete representation of the feasible space of solutions in a predefined period of time.

The visual representation of the flexibility space is not straightforward. In fact, when planning for more than three time steps ahead it becomes impossible to visually represent that type of information. It should be stressed that there is a difference between visually representing the hourly limits of the flexibility band (like the flexibility envelope in [11]) and the actual visual representation of such feasible domain. For illustration, let assume a single electric battery with $6.4 \mathrm{kWh}$ of storage capacity, maximum charge/discharge power of $3.3 \mathrm{~kW}$, initial SoC in $1.28 \mathrm{kWh}(20 \%)$ limited to $0.96 \mathrm{kWh}(15 \%)$. Fig. 1 represents the hourly limits of the flexibility band that this battery can provided considering hourly time steps and the referred initial state.

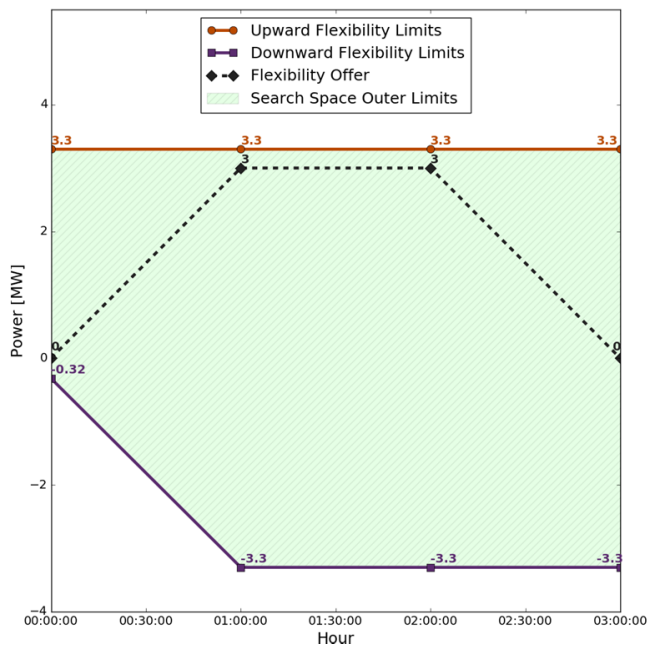

Figure 1. Hourly limits for flexibility search space.

Fig. 1 must be interpreted as an hourly limit for flexibility provision by the battery, i.e., for hour 00:00 the maximum energy that the battery can accommodate is $3.3 \mathrm{kWh}$ as it only leads to $4.58 \mathrm{kWh}$ SoC considering the $1.28 \mathrm{kWh}$ initial SoC. On the other hand, as the minimum allowed SoC is $0.96 \mathrm{kWh}$, the battery will only be able of providing $0.32 \mathrm{kWh}$ of upward flexibility. From hour 01:00 onwards the hourly maximum and minimum flexibility provision limits equal the maximum and minimum charging and discharging limits, as it is always 
possible to select a feasible trajectory that in any of those hours is able of providing such flexibility without violating the SoC constraints. With that said, one can not imply that any trajectory composed by hourly values within the domain defined by those limits can be considered feasible. If, for instance, a trajectory composed by the hourly flexibility offers expressed in $\mathrm{kW}[0.0,3.0,3.0,0.0]$ and presented in Fig. 1 is selected, the correspondent SoC (in $\mathrm{kWh}$ ) would be [1.28, $4.28,7.28,7.28]$, which is not feasible from hour 02:00 onwards. Concluding, it is possible to visually represent the theoretical hourly flexibility provision limits, although these limits do not defined the domain of feasible flexibility provision that is the focus of this study. Unfortunately, the domain studied in this work cannot be visually represented for more than three time-steps decision problems, as it becomes at least a four-dimension representation.

\section{B. Modelling of the Flexibility Set}

By means of sampling routines using domain knowledge, a sufficient number of feasible trajectories can be built to define the flexibility (or feasible) space. After constructing a considerable number of feasible trajectories the flexibility provision search space that can be provided by the HEMS to the DSO or demand/flexibility aggregator can be delimited and defined by means of a Support Vector Data Description (SVDD) function. In this study a One-Class Support Vector Machine (SVM) function is used for the feasibility detection [15]. The use of SVDD was inspired by the methodology described in [16] for VPP application. This function is typically used for novelty detection where a given set of samples are provided to the function and a model is built by detecting the soft boundary of that set. The originated model is capable of classifying new points as belonging to that set or not. Fig. 2 represents the block-diagram of the proposed methodology. In the first block of the diagram are represented the two stages of the algorithm responsible to build feasible trajectories, which must respect technical and costumer's preferences constraints. Customers' preferences can be embedded into the proposed algorithm by modelling, per example, the desire of maximizing the PV generation consumption, which translates in using the most of the storage unit capacity to storage PV generation in times of PV surplus; or by pre-defining the hot water usage that the customer wants to assure is available at certain times of the day. Those feasible trajectories are then used as input in the second block where the learning of the feasibility domain occurs by means of the previously referred One-Class SVM function. At the end, represented in the third diagram block, the DSO uses the model created to validate flexibility set-points to send to HEMS that satisfy the flexibility needs that were previously identified. In order to assess the flexibility provision potential, the DSO, or aggregator, only needs to receive from its flexible assets (HEMS in this study) the support vectors identified by the One-Class SVM function. This way, all the HEMs equipment and problem formulation regarding the HEMS internal operation constraints does not need to be accounted by the
DSO in its optimization software, reducing computational effort and time.

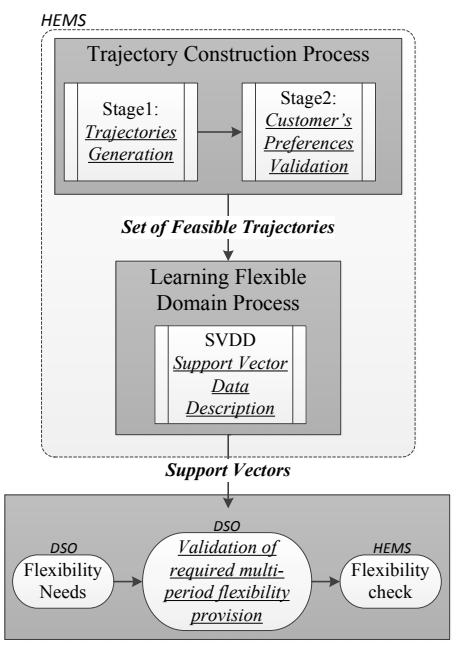

Figure 2. Algorithm block-diagram.

\section{Generation of feasible trajectories}

In order to evaluate whether or not a trajectory is feasible, one must account for four variables: a decision variable which represents the power flow in the battery's inverter, Pbat, (in this study a domestic electric battery was considered as electric storage), a decision variable regarding the operating status of the EWH, Pewh, a state variable that represents the SoC of the storage equipment, and another state variable representing the temperature of the water inside the tank of the EWH.

The developed trajectory construction algorithm has two stages. In the first stage a random sampling routine is used to create the trajectories, which is able of providing diversity in the set of trajectories built. The constructed trajectory, traj, will be the result of the sum of the decision variables for all the time steps considered, following (1). For each 15 minute time step a random value ranged between the minimum and maximum battery power is chosen and the decision of turning on, or not, the EWH is made. Accordingly, the flexibility to be provided by the HEMS is limited to the battery's charging and discharge powers (Pbat ${ }^{\max }$ and $\mathrm{Pbat}^{\text {min }}$ ) and the EWH nominal power $\left(P e w h^{n o m}\right),(2)$ and (3) respectively. Afterwards, based on the conditions of the previous period, the battery SoC and the water temperature of the EWH are updated and one must assure that the values of correspondent decision variable do not lead to impractical battery's state of charge (4) and (5). In case of constraint violation of the SoC limits, the decision variable is modified so that the $\mathrm{SoC}$ in the current time step is considered viable.

Regarding the state variable related to the temperature of the water tank, $\theta$, one must assure that a pre-defined temperature range is permanently guaranteed (6). If a violation of the water temperature range constraint occurs, the decision variable for the operating status of the EWH is changed in one 
of two ways: if the original decision was to keep the EWH "off" leading to temperatures below the pre-defined minimum, then the new decision is to turn "on" the EWH for that time step; on the other hand, if the maximum temperature was reached as the original decision was to have the EWH with the "on" status, then the decision is changed to the "off" status. If at a certain time step, changing the EWH operating status is not enough to comply with the temperature constraint the algorithm automatically rejects the so far constructed trajectory and proceeds to the construction of the next trajectory. Equation (7) represents the water temperature variation during time, dependent on the volume of hot water used and Pewh. The physically-based load model adopted for the EWH modeling is aligned with the one used in [17].

In the second stage of the algorithm, the customer's defined preferences are evaluated, which for this study refers to the use of the battery to accommodate the PV generation surplus. In this study we used a 24 hours planning period with 15 minutes resolution. The evaluation function developed for this second stage makes use of both the decision and state variables. There is a need to identify the time steps where the PV generation surpasses the house's static demand and to quantify by how much it does it. Then, for each time step, the developed function updates the state variable related to the battery state of charge if that time step had been pointed as one where there is PV surplus. This PV generation surplus is used to charge the battery and consequently increasing the SoC, taking into account the battery's efficiency and restricted to its maximum charging power (8). Accordingly, (4) and (5) give place to (9) and (10). Basically, the maximum and physically possible amount of PV surplus energy that the battery can absorb without being used for flexibility provision must still be assured when defining the feasible trajectories for flexibility provision.

$$
\begin{aligned}
& \operatorname{traj}_{h}=\mathrm{Pbat}_{h}+\mathrm{Pewh}_{h} \\
& \text { Pbat }^{\text {min }} \leq \text { Pbat }_{h} \leq \text { Pbat }{ }^{\max } \\
& \text { Pewh }_{h}= \begin{cases}0 & , \text { for off status } \\
\text { Pewh } & \text {, for on status }\end{cases} \\
& \text { SoC } C^{\text {ini }}+\sum_{h=1}^{H} \text { Pbat }_{h} \leq \text { SoC } \max \\
& \text { SoC }{ }^{\text {ini }}+\sum_{h=1}^{H} \text { Pbat }_{h} \geq \text { SoC }^{\text {min }} \\
& \theta^{\min } \leq \theta_{h} \leq \theta^{\max } \\
& \theta_{h}=\theta_{h-1}+ \\
& \frac{\Delta t}{C}\left[-\alpha\left(\theta_{h-1}-\theta_{\text {house }}\right)-c_{p} v_{h}\left(\theta_{\text {des }}-\theta_{\text {inl }}\right)+P e w h_{h}\right] \\
& P b a t_{h}+P V_{h}^{\text {sur }} \leq P b a t^{\max }, \forall h \\
& \text { SoC }{ }^{\text {ini }}+\sum_{h=1}^{H} \text { Pbat }_{h}+P V_{h}^{\text {sur }} \leq S o C^{\max }
\end{aligned}
$$

$$
\text { SoC } C^{\text {ini }}+\sum_{h=1}^{H} \text { Pbat }_{h}+P V_{h}^{\text {sur }} \geq \text { SoC }^{\text {min }}
$$

In (7), $\Delta t$ is the time step [h], $\mathrm{C}$ is the thermal capacity $\left[\mathrm{kWh} /{ }^{\circ} \mathrm{C}\right]$ set to $0.117, \alpha$ is the thermal admittance $\left[\mathrm{kW} /{ }^{\circ} \mathrm{C}\right]$ set to $-9.42^{-4}, \theta_{\text {house }}$ is the house indoor temperature set to $20^{\circ} \mathrm{C}$, $c_{p}$ is the water specific heat $\left[\mathrm{kWh} /\left(\operatorname{ltr} .{ }^{\circ} \mathrm{C}\right)\right], v_{h}$ is the hot water consumption at time $h, \theta_{\text {des }}$ is the desired temperature for water consumption, and $\theta_{i n l}$ is the inlet water temperature.

Fig. 3 represents the home's load and forecasted PV generation profiles (top) and the $\mathrm{SoC}$ variations before and after this second stage of feasibility evaluation. As one can verify from the figure analysis, the final $\mathrm{SoC}$ variation maintains the shape of the initial SoC variation but comes shifted up by the amount of PV energy surplus accumulated until each time step.
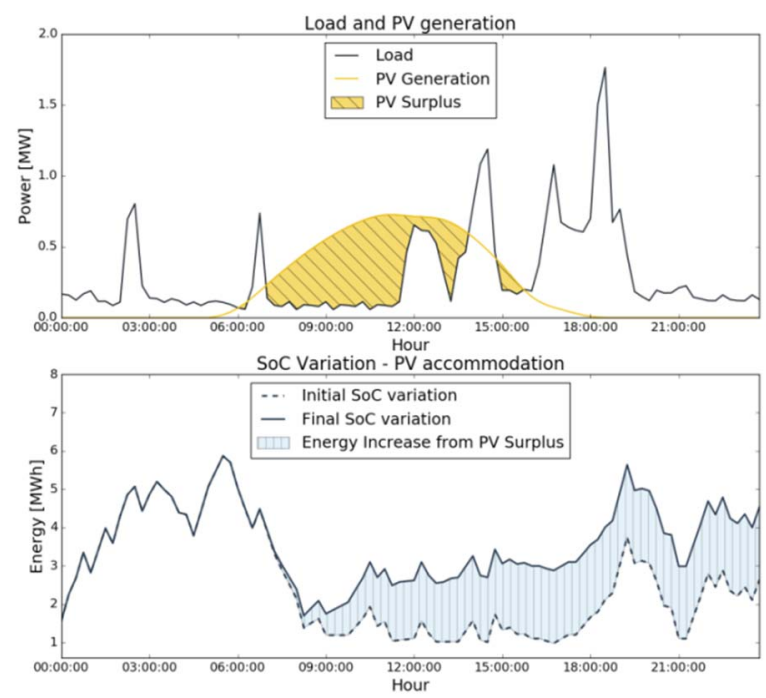

Figure 3. Load and PV generation profiles (top); SoC variation due to PV Surplus (bottom).

\section{Black-box model for the HEMS flexibility}

Having constructed a sufficient large number of feasible trajectories with the method described in section III.C, the search space for the flexibility provision can be defined. To do so, those trajectories are used as input in a Support Vector Data Description function, namely a One-Class Support Vector Machine function available in the Scikit-Learn Python Library ${ }^{1}$. The model that is created by this function is able of delimiting and learning the feasibility boundary (or flexibility set) based on the input data. The model identifies the minimum number and the actual support vectors that describe the highdimension sphere representing the feasible domain and, applying (11), is able of classifying trajectories as feasible or not. Accordingly, it compares the radius in the high dimension domain that the trajectory represents and compares it to the defined radius of the referred sphere. If the radius of the

\footnotetext{
${ }^{1}$ http://scikit-learn.org/
} 
trajectory evaluated is equal or inferior to the sphere's radius, then the trajectory is classified as feasible, as it falls into the feasibility domain. Equation (11) represents the formula to calculate the radius, where $x_{i}$ and $x_{j}$ are support vectors and $x$ is the trajectory being evaluated. Deeper information regarding this methodology can be consulted in [16].

$$
R^{2}(x)=1-2 \sum_{i} \beta_{i} k\left(x_{i}, x\right)+\sum_{i, j} \beta_{i} \beta_{j} k\left(x_{i}, x_{j}\right)
$$

\section{CASE STUDY}

The proposed algorithm was used to validate the dispatch of the flexible resources of a MG when operating islanded. The considered MG algorithm schedules an emergency operational plan for the next hours, based on load and microgeneration forecasting, considering the possibility of an unplanned islanding. The plan consists in set-points for the $\mathrm{MG}$ controllable storage unit(s) providing secondary frequency regulation, considering its power and energy capacity. The main objective is to maximize the energy capacity of the grid forming storage unit(s) providing frequency and voltage regulation. When the MG power and energy reserve capacity is not sufficient to supply the loads, it will be necessary to explore the flexibility available at residential level. Based on an unbalanced power flow studies, the HEMS flexibility will be mobilized in the flexible nodes which are in the phase and feeder with the highest voltage deviation, being the active power dispatch of each HEMS validated by the black-box model for the HEMS flexibility, presented in III.D. Regarding the HEMS problem formulation, in (7) the desired temperature for water consumption, $\theta_{\text {des }}$, is set to $38^{\circ} \mathrm{C}$, and the inlet water temperature, $\theta_{\text {inl }}$, is set to $17^{\circ} \mathrm{C}$.

The considered MG emergency dispatch strategy was defined for a LV network endowed with three storage devices, where one provides primary frequency and voltage regulation (VSI) while the other two are current controlled (PQ units). The grid forming storage unit has a $60 \mathrm{~kW} / 30 \mathrm{kWh}$ capacity and the other two have $10 \mathrm{~kW} / 10 \mathrm{kWh}$ capacity. It is assumed that some LV clients have installed microgeneration such as PV panels. An illustrative time horizon of two hours with 15 min discretization was considered, corresponding to the maximum time the $\mathrm{MG}$ was initially planned to operate autonomously.

The islanding event occurs in the beginning of the simulation $(\mathrm{t}=0 \mathrm{~min})$. Before the islanding the $\mathrm{MG}$ storage devices SOC is respectively: $\mathrm{VSI}=70 \%, \mathrm{PQ} 1=20 \%$ and PQ2 $=10 \%$. Considering the initial SOC, load, and microgeneration forecasts the dispatch strategy first defines the operational plan for the distributed storage devices. However, since the VSI hasn't enough energy capacity to supply the MG loads during the two hours, the algorithm needs to mobilize flexibility. The active power requested to the HEMS connected to the MG is presented in Fig. 4. As shown in Fig. 5 without flexibility provision the VSI will fully discharge in the beginning of the second hour, meaning that the MG would blackout. However, if the HEMS flexibility is considered it is possible to maintain the VSI SOC above $20 \%$.

Currently, the efficiency assessment on the classification of new trajectories by the developed algorithm only considers as efficiency control set the feasible trajectories used as input for the One-Class SVM function. In future work, new feasible and non-feasible trajectory sets will be created for a more profoundly acknowledge of the algorithm efficiency. Basically, it is expected that the algorithm performs well not only on correctly identifying feasible trajectories, but also on correctly identifying non-feasible ones.

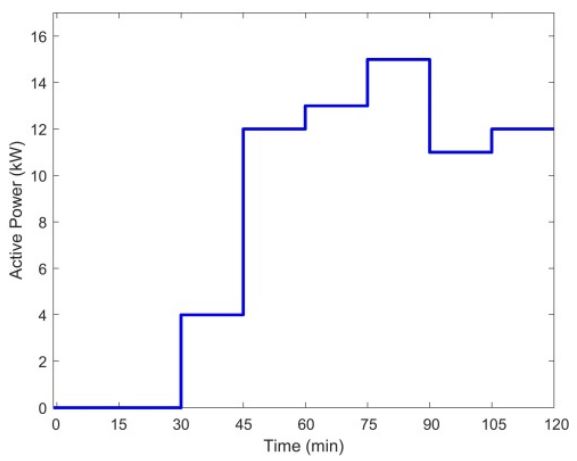

Figure 4. Active power injected by the flexible controllable resources.

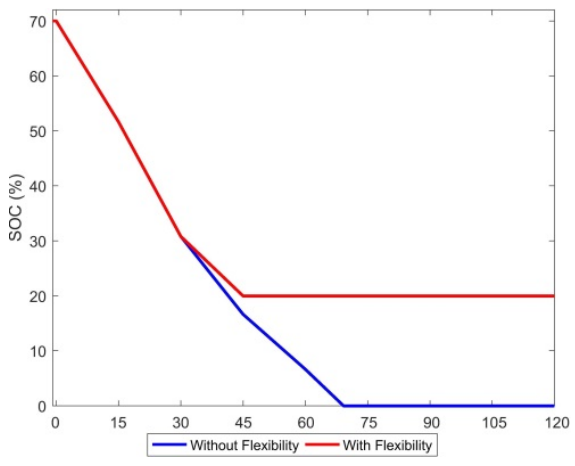

Figure 5. VSI State-of-Charge variation.

Depending on the type of samples that are provided to the function, the One-Class SVM function hyper-parameters, such as the kernel type that is used in the embedded algorithm or even the gamma kernel coefficient that is used $(\gamma)$ must be properly defined. By experience it was found that the most suitable type of kernel for this study is the Sigmoid. Additionally, the pre-defined value for the $\gamma$ and $n u$ parameters can influence the efficiency of the created models. The $n u$ parameter refers to an upper bound on the fraction of training errors and a lower bound of the fraction of support vectors. Results show that the Sigmoid kernel based model performs better that the others for $\gamma=0.05$ and $n u=0.001$. The evaluations were made on the original set of samples (feasible trajectories) used as input for the model construction. For these conditions, the error is of $1.3 \%$, which results from the number 
of wrongly rejected trajectories out of the total number of input samples.

After defining the function parameters that better fit the type of samples that are used, the resulting model is prepared to evaluate trajectories on being feasible or not. This kind of features can be used by the DSO when performing grid operational assessment regarding possible provision of flexibility in LV distribution grid in case of detecting local constraints in such grids. Receiving the constructed model from each HEMS, the DSO has means to understand and quantify the expected flexibility to be provided by them without having to know or model the home's storage and microgeneration equipment, demand patterns and customer's preferences, avoiding confidentiality problems and saving computational time. Similarly, demand/flexibility aggregators can also use the trained models provided by the HEMSs to prepare optimally computed offers to bid in the respective markets.

\section{CONCLUSION}

The use of reliable and smartly gathered information regarding the flexibility provision that can come from HEMS is of great value for DSOs and aggregators as market agents. Nevertheless, it is not a trivial task to efficiently delimited the HEMS multi-temporal flexibility feasible domain, especially when simultaneously considering microgeneration like domestic PV generation, energy storage equipment, EWH and costumer preferences regarding comfort constraints and operation strategy for its energy storage equipment. The proposed algorithm is able of efficiently learn and define the feasibility search space endowing DSOs and aggregators of a tool that, in a reliable and time efficient fashion, provides them valuable information. This information is essential for these agents to understand the fully grid operation and economic benefits that can arise from the smart management of their flexible assets. The algorithm shows very low errors when identifying the feasibility of multi-temporal flexibility offers.

Uncertainty is becoming an indispensable topic in the operation of electric power systems, particularly at the LV and MV distribution grid level. Accordingly, a future version of this algorithm is already being planned in order to produce robust trajectories (i.e., feasible for diverse PV generation scenarios), using them as backbone in the construction of the flexibility search space to be provided to DSO or demand/flexibility aggregators.

\section{ACKNOWLEDGEMENTS}

This work is funded by SENSIBLE project (Storage ENabled SustaInable energy for BuiLdings and communitiEs www.h2020-projectsensible.eu) that has received funding from the European Union's Horizon 2020 - The EU Framework Programme for Research and Innovation 2014-2020, under grant agreement No.[645963]. The work was also supported in part by Fundação para a Ciência e a Tecnologia (FCT) under Grant SFRH/BD/117428/2016.

\section{REFERENCES}

[1] M. Hasheminamin, V. G. Agelidis, and A. Heidari, "Impact study of high PV penetration in low and medium-voltage networks when considering residential and industrial load profile," in Proceedings of 2013 International Conference on Renewable Energy Research and Applications, ICRERA 2013, 2013, pp. 347-352.

[2] P. Lazzeroni, S. Olivero, F. Stirano, and M. Repetto, "Impact of PV penetration in a distribution grid: A Middle-East study case," in 2015 IEEE 1st International Forum on Research and Technologies for Society and Industry, RTSI 2015 - Proceedings, 2015, pp. 353-358.

[3] J. Vasiljevska, J.A. Peças Lopes, M.A. Matos, "Integrated microgeneration, load and energy storage control functionality under the multi micro-grid concept," Electric Power Systems Research, vol. 95, Feb. 2013, pp. 292-301

[4] N. Silva, P. M. Silva, L. Seca, A. Madureira, J. Pereira, F. Melo, "LV SCADA - How to Effectively Manage LV Networks with Limited Topology and Electrical Characteristics Data," CIRED 2015 - The 23rd International Conference and Exhibition on Electricity Distribution, Lyon, France, June, 2015.

[5] J. R. Snape, B. M. Ardestani, and P. Boait, "Accommodating renewable generation through an aggregator-focused method for inducing demand side response from electricity consumers," IET Renewable and Power Generation., vol. 7, no. 6, pp. 689-699, Nov. 2013.

[6] L. Gkatzikis, I. Koutsopoulos, and T. Salonidis, "The role of aggregators in smart grid demand response markets," IEEE J. Sel. Areas Commun., vol. 31, no. 7, pp. 1247-1257, Jul. 2013.

[7] M. Gonzalez Vaya and G. Andersson, "Optimal Bidding Strategy of a Plug-In Electric Vehicle Aggregator in Day-Ahead Electricity Markets Under Uncertainty," IEEE Trans. Power Syst., vol. 30, no. 5, pp. 23752385, Sep. 2014

[8] M. Marques, P. Mousinho, A. Leiria, R.J. Bessa, C. Gouveia, A. Madureira, C. Moreira, M. Gerlich, S. Rodriguez, "Integration of energy storage in LV Grid normal and emergency operation," CIRED 2016 Workshop, Helsinki, Finland, June 2016.

[9] L. Zhao, W. Zhang, H. Hao, and K. Kalsi, "A Geometric Approach to Virtual Battery Modeling of Thermostatically Controlled Loads," arXiv: $1608.04422 \mathrm{v} 1$.

[10] E. Polymeneas and S. Meliopoulos, "Aggregate modeling of distribution systems for multi-period OPF," in 2016 Power Systems Computation Conference (PSCC), 2016, pp. 1-8.

[11] M. A. Bucher, S. Delikaraoglou, K. Heussen, P. Pinson, and G. Andersson, "On quantification of flexibility in power systems," in 2015 IEEE Eindhoven PowerTech, 2015, pp. 1-6.

[12] T. Nuytten, B. Claessens, K. Paredis, J. Van Bael, and D. Six, "Flexibility of a combined heat and power system with thermal energy storage for district heating," Appl. Energy, vol. 104, pp. 583-591, 2013.

[13] H. Nosair and F. Bouffard, "Flexibility Envelopes for Power System Operational Planning," IEEE Trans. Sustain. Energy, vol. 6, no. 3, pp. 800-809, Jul. 2015.

[14] J. Zhao, T. Zheng, and E. Litvinov, "A Unified Framework for Defining and Measuring Flexibility in Power System," IEEE Trans. Power Syst., vol. 31, no. 1, pp. 339-347, Jan. 2016.

[15] Pedrogosa et al., "Scikit-learn: Machine Learning in Python," JMLR 12, pp. 2825-2830, Oct. 2011

[16] J. Bremer, B. Rapp, M. Sonnenschein, "Encoding distributed search spaces for virtual power plants," 2011 IEEE Symposium on Computational Intelligence Applications Smart Grid, pp. 1-8, April 2011.

[17] M. Heleno, M. A. Matos, J. P. Lopes, "Availability of termal loads to provide reserve services," in 2013 IEEE Grenoble PowerTech, pp. 1-6, June 2013. 